\title{
TIMELESS inhibits breast cancer cell invasion and metastasis by down-regulating the expression of MMP9
}

\author{
Bowen Li ${ }^{\dagger}$, Liying $\mathrm{Mu}^{\dagger}$, Yanan Li, Kangkai Xia, Yuxi Yang, Sattout Aman, Bashir Ahmad, Shujing Li \\ and Huijian Wu* ${ }^{*}$
}

\begin{abstract}
Breast cancer is the first killer leading to female death, and tumor metastasis is one of the important factors leading to the death of patients, but the specific mechanism of breast cancer metastasis is not very clear at present. Our study showed that overexpression of TIMELESS could significantly inhibit the invasion and metastasis of breast cancer cells ZR-75-30 and the assembly of F-actin protein. On the contrary, knockdown of TIMELESS promoted the invasion and metastasis of breast cancer cells. Further study revealed that TIMELESS overexpression decreased the mRNA and protein levels of MMP9. Furthermore, TIMELESS could interact with p65, leading to repress the association of p65 and its acetyltransferase CBP and down-regulating the acetylation level of p65, which inhibited the activation of NF-KB signal pathway. In conclusion, our research showed that TIMELESS may repress the invasion and metastasis of breast cancer cells via inhibiting the acetylation of p65, inhibiting the activation of NF-kB, thus down-regulating the expression of MMP9, and then inhibiting the invasion and metastasis of breast cancer cells.
\end{abstract}

Keywords: TIMELESS, MMP9, Invasion, Metastasis, Breast cancer

\section{Background}

Breast cancer is one of the most serious threats to women's health worldwide. Its annual average growth rate is about $4 \%$ with poor prognosis and the median survival time is only 2-3 years in China, which is a bad situation and closely related to the invasion and metastasis of breast cancer cells $[1,2]$. However, the molecular mechanism is not yet clearly demonstrated. Therefore, research on the mechanisms of breast cancer invasion and metastasis will help provide new strategies for clinical diagnosis and treatment.

In the past, cancer research mainly focused on the mutation of cancer cells to gain the advantage of cell

\footnotetext{
*Correspondence: Isj@dlut.edu.cn; wuhj@dlut.edu.cn

${ }^{\dagger}$ Bowen Li and Liying Mu contributed equally to this work

School of Bioengineering \& Key Laboratory of Protein Modification and Disease, Liaoning Province, Dalian University of Technology, 2 Ling Gong Road, Dalian 116024, Liaoning, China
}

proliferation or survival. At present, more and more evidence show that tumor microenvironment, especially extracellular matrix, has become the main factor affecting the progress of cancer. MMPs are expressed in almost all human cancers [3], such as normal fibroblasts, cancer-related fibroblasts and cancer cells themselves, which can be expressed in the adjacent stroma of tumors. Because MMPs can promote angiogenesis, tumor growth and metastasis, tumor immune regulation and affect the microenvironment before tumor metastasis, the expression of MMP family is closely related to tumor invasion, stage and prognosis [4].

MMP9 is one of the most important members of MMPs family and one of the target genes of NF-kB. It is also called gelatinase B. It can degrade collagen IV, the main component of the cell basement membrane, which plays an important role in cancer metastasis [5]. MMP9 can take different effects in the process of cell scatter, such as 
tumor invasion, tumor-induced angiogenesis, immune regulation of tumor microenvironment and the formation of microenvironment before metastasis to promote tumor cell distant metastasis [6]. The increased expression of MMP9 is also related to the invasion, metastasis and poor prognosis of different types of cancer, such as cervical cancer, colorectal cancer, ovarian cancer and breast cancer [7-10].

The expression of MMP9 is a rate-limiting step of MMP9 expression. There are many transcriptional factors that can bind with MMP9 promoter, including NF-kB, AP1, SP1, etc. [11]. Therefore, the study of the transcriptional regulation of MMP9 will be favorite to revealing its fine mechanism of action in tumor.

TIMELESS was originally identified in Drosophila melanogaster as an integral part of the circadian rhythm [12]. In mammals, TIMELESS still has the function of regulating daily rhythm, but TIMELESS is best characterized in DNA replication and damage repair $[13,14]$. In a normal environment, TIMELESS can ensure the normal replication of DNA. This important role is mainly achieved by controlling DNA replication, maintaining the stability of replication fork and genome $[15,16]$. Therefore, TIMELESS is considered to be a member of the replication fork protection complex. In recent years, it has been reported that TIMELESS can interact with PARP-1 to promote homologous recombination repair of DNA double-strand. This process neither depends on poly ADP ribosylation nor affects the enzyme activity of PARP1. PARP-1 mediates the recruitment of TIMELESS into DSB (DNA double-strand breaks) to promote homologous recombination repair $[17,18]$

In our study, TIMELESS inhibited the invasion and metastasis of breast cancer cells by down-regulating the mRNA and protein levels of MMP9. Since MMP9 is a target gene of the NF-kB signaling pathway, whether TIMELESS down-regulates MMP9 is examined through the NF- $\mathrm{KB}$ signaling pathway. The study of TIMELESS on the NF- $k B$ signal pathway showed that TIMELESS could inhibit the interaction between NF- $\mathrm{kB}$ p 65 and its acetyltransferase CBP through the interaction with p65, thereby reducing the acetylation of p65, thus impeding the activation of NF- $\mathrm{kB}$ signal pathway.

\section{Materials and methods}

\section{Cell culture and transfection}

HEK-293T, COS-7, ZR-75-30, T47D and MCF-7 cells used in this experiment were all generous gifts from the Cell Resource Center of Dalian Medical University and they are cultured as previously described [19]. Cells were grown at $37{ }^{\circ} \mathrm{C}$ in a humidified $5 \% \mathrm{CO}_{2}$ atmosphere and then the cells were transfected with the appropriate plasmids using Lipofectamine 2000.

\section{Plasmid construction and antibodies}

Human TIMELESS was cloned from a pCMV-Timeless plasmid. The sense and antisense primers were TIMELESS-F: 5'-CCAAGCTTGGGATGGACTTGC ACATGATGAAC- $3^{\prime}$ and TIMELESS-R: $5^{\prime}$-GCTCTA GAGCTCAGTCATCCTCATCATCCTC-3', respectively. The prepared TIMELESS DNA fragment was then inserted into the expression vector $3 \times$ FLAGpcDNA3.1 at the Xba I-Hind III sites. pGL3-MMP9-luc was constructed in my lab before and they both were acquired as previously described [20]. Mouse antiFlag antibody was purchased from Sigma (M2, 1:8000). Rabbit anti-Timeless was purchased from Abcam (ab72458, 1:6000). Rabbit anti-MMP9 antibodies were purchased from Wanlei (WL02141, 1:500). Rabbit p65 antibody was obtained from Abcam (ab16502, 1:5000). Rabbit GFP antibody was obtained from GeneTex (GTX113617, 1:8000). Mouse anti $\beta$-Tublin was purchased from ORIGENE (TA347065, 1:6000). Mouse anti GAPDH was purchased from ORIGENE (TA802519, 1:6000). Rabbit acetylated lysine antibody was obtained from CST (9441, 1:3000).

\section{Transwell and scratch wound-healing assays}

Transwell and scratch wound-healing were performed to access the properties of invasion and metastasis of breast cancer cells and they were operated as previously described [21]. The inoculation amount of the cells in one chamber in the Transwell migration experiment was $2 \times 10^{4}$ and that was $5 \times 10^{4}$ in the Transwell invasion experiment.

\section{Luciferase reporter assay}

Promoter activity was examined by a luciferase assay system. Cells were inoculated in 24-well plates, and cultured for $24 \mathrm{~h}$. Next, the cells were transfected with corresponding plasmids using Lipofectamine 2000 according to the company's specification. Twenty-four hours following the transfection, the cells were subjected to luciferase and Renilla activity assays according to the manufacturer's instructions (Promega, Madison, WI, USA).

\section{Construction of the shTIMELESS\#1 and ShTIMELESS\#2}

TIMELESS is expressed at various levels in different breast cancer tissues. Thus, carrying out a knockdown experiment with a plasmid expression vector of RNA interference targeting TIMELESS is necessary. The pRNAT-U6.1 vector was purchased from GenScript (GenScript, Piscataway, NJ, USA). The sequences of RNA interference were as follows: 5'-GGTTCGAGAGATGAC 
TGAGGGCTAT-3' (shTIMELESS\# 1 ) and $5^{\prime}$-TCCAGG GTAGCTTAGTCCTTTCAAA-3' (shTIMELESS\#2) [24].

\section{Realtime PCR}

The extraction of total RNA and real-time PCR were performed as previously described [22]. The primers for TNF- $\alpha$ mRNA were CCCAGGCAGTCAGATCAT CTTC (forward) and AGCTGCCCCTCAGCTTGA (reverse); VEGFA mRNA were CGGGAACCAGATCTC TCACC (forward) and AAAATGGCGAATCCAATT CC (reverse); uPA mRNA were AGTGTCAGCAGC CCCACT (forward) and CCCCCTGAGTCTCCCTGG (reverse); MMP9 mRNA were TACTGTGCCTTTGAG TCCG (forward) and TTGTCGGCGATAAGGAAG (reverse); TIMELESS mRNA were CTCCTCCGGGCT TCTGA (forward) and CCATACATCAGTGGACCA ACC (reverse); and glyceraldehyde 3-phosphate dehydrogenase (GAPDH as a reference gene) mRNA were GGG TGTGAACCATGAGAAGT (forward) and GACTGT GGTCATGAGTCCT (reverse). The mRNA level was normalized to GAPDH as the endogenous control. Each target was measured in triplicate.

\section{The mammalian two-hybrid system}

The checkmateTM mammalian two-hybrid system was purchased from Promega. p65 and TIMELESS were subcloned into pBIND at EcoRV/BamHI sites and pACT at Mlu site, respectively.

\section{Immunoprecipitation and Western blot assay}

Western blot assays were conducted as previously described [23].

\section{Immunofluorescence assay}

Immunofluorescence assays were conducted as previously described [23].

\section{Statistical analysis}

Data was examined as means \pm SDs. An unpaired t-test was used when the results from the two groups were compared. Statistical analysis was carried out by oneway analysis of variance with Bonferroni's multiple-comparison correction for comparison among three or more groups. Statistical significance was considered at the $\mathrm{P}<0.05$ level.

\section{Results}

TIMELESS down-regulates the migration and invasion of breast cancer cells

Based on the results of KM analysis on patients of basallike breast cancer, the survival of patients with high expression of TIMELESS was significantly higher than that of patients with low expression $(\mathrm{P}<0.05)$, suggesting that the expression of TIMELESS was positively correlated with the survival of patients with basal-like breast cancer (Additional file 1: Figure S1). Basal-like morphology was more often associated with metastatic breast cancer [24]. Three different breast cancer cell morphology was examined and the result showed that in the cell lines with high TIMELESS expression, the cells were mainly epithelioid morphology (Fig. 1a, b), while in ZR-75-30 cell line, the expression of TIMELESS was lower than the other two, and the cell morphology was mesenchymal-like (Fig. 1a, b). This suggested that TIMELESS might play an important role in the invasion and metastasis of breast cancer cells. In ZR-7530 cells, TIMELESS overexpression could significantly inhibit the speed of scratch healing (Fig. 1c). On the contrary, TIMELESS knockdown accelerated this process in T47D cells (Fig. 1d). Similar results were obtained from the following invasion and metastasis experiments. The overexpression of TIMELESS repressed the invasion and metastasis of breast cancer cells (Fig. 1e), while down-regulating TIMELESS facilitated the invasion and metastasis of breast cancer cells (Fig. 1f). The assembly of intracellular actin into $\mathrm{F}$-actin, a process having an important impact on cell characteristics of motility and invasiveness. Specifically, the cell polarity is reduced or lost and the relatively rounded cells change into a long and narrow shape with F-actin organized along the direction of cell movement, thereby facilitating cell migration from the fixed surface to the free surface [25]. Immunofluorescence experiments showed that the organization of actin was disrupted and not easily integrated into bundles of F-actin with TIMELESS overexpression compared to that of the control group (Fig. 1g). While silencing TIMELESS, F-actin was organized in abundant stressed fibers (Fig. 1h), i.e., overexpression of TIMELESS in breast cancer cells inhibited the organization of loose actin into F-actin while TIMELESS knocking down promoted the assembly process of actin. The role of TIMELESS on the motility of breast cancer cells was not owing to impairing cell proliferation, because the overexpression or knockdown of TIMELESS had no significant effect on cell proliferation within $24 \mathrm{~h}$ (Additional file 2: Figure S2 and Additional file 3: Figure S3). The knockdown efficiency of TIMELESS was shown in Additional file 3: Figure S3. To sum up, overexpression of TIMELESS inhibited the invasion and metastasis of breast cancer cells while knocking down TIMELESS promoted these two abilities.

\section{TIMELESS inhibited the expression of MMP9}

MMP9 is a key factor in the process of tumor invasion and metastasis. Next, we tested whether TIMELESS 


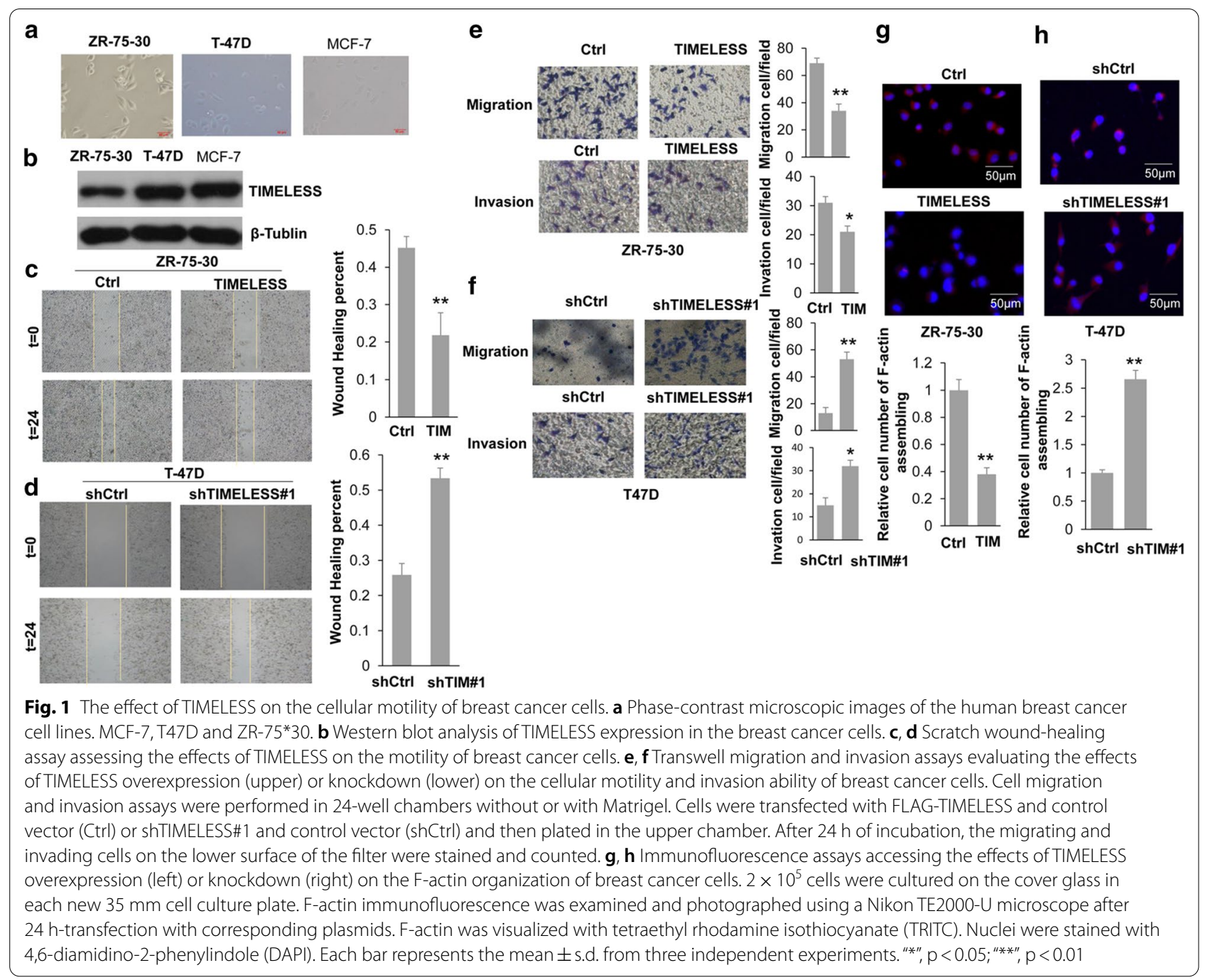

could inhibit the invasion and metastasis of breast cancer via The MMP9 signaling pathway. Through the luciferase reporter gene experiment, overexpression of TIMELESS in different cell lines could reduce the transcriptional activity of MMP9 (Fig. 2a), and this inhibition is dose-dependent (Fig. 2b). The mRNA level of MMP9 was also detected and the result showed that the overexpression of TIMELESS decreased the mRNA level of MMP9 (Fig. 2c), on the contrary, TIMELESS knockdown increased the expression of MMP9 (Fig. 2d). Similar results were obtained in the examination of MMP9 protein level in breast cancer cells, that is, the overexpression of TIMELESS increased the expression of endogenous MMP9 (Fig. 2e), while TIMELESS knockdown inhibited the protein level of MMP9 (Fig. 2f). It can be concluded that TIMELESS may negatively regulate the mRNA and protein levels of MMP9.

\section{TIMELESS repressed MMP9 expression by reducing} the activation of NF-KB

As we all know, MMP9 is an important downstream target gene of the NF- $\mathrm{kB}$ signaling pathway, so we studied whether TIMELESS regulates MMP9 through the NF- $\mathrm{kB}$ signaling pathway. First, the effect of TIMELESS on the activation of the NF- $\mathrm{kB}$ signaling pathway was examined. Results as shown in Fig. 3a, p65 overexpression alone could activate the NF- $\mathrm{KB}$ signal pathway, while overexpression of both p65 and TIMELESS significantly reduced NF- $\mathrm{kB}$ reporter gene activity compared with p65 overexpression alone (Fig. 3a). By analyzing the mRNA levels of IL8, uPA and TNF $\alpha$ which are the target genes of NF- $\mathrm{B}$ signal pathway, TIMELESS overexpression downregulated the mRNA levels of NF- $\mathrm{B}$ downstream target genes (Fig. 3b). To determine whether TIMELESS regulated the expression of MMP9 through NF- $\kappa B$, we constructed another two MMP9 luciferase reporter gene, NF-kB-del-MMP9-luci (deletion of NF- $\mathrm{BB}$ 


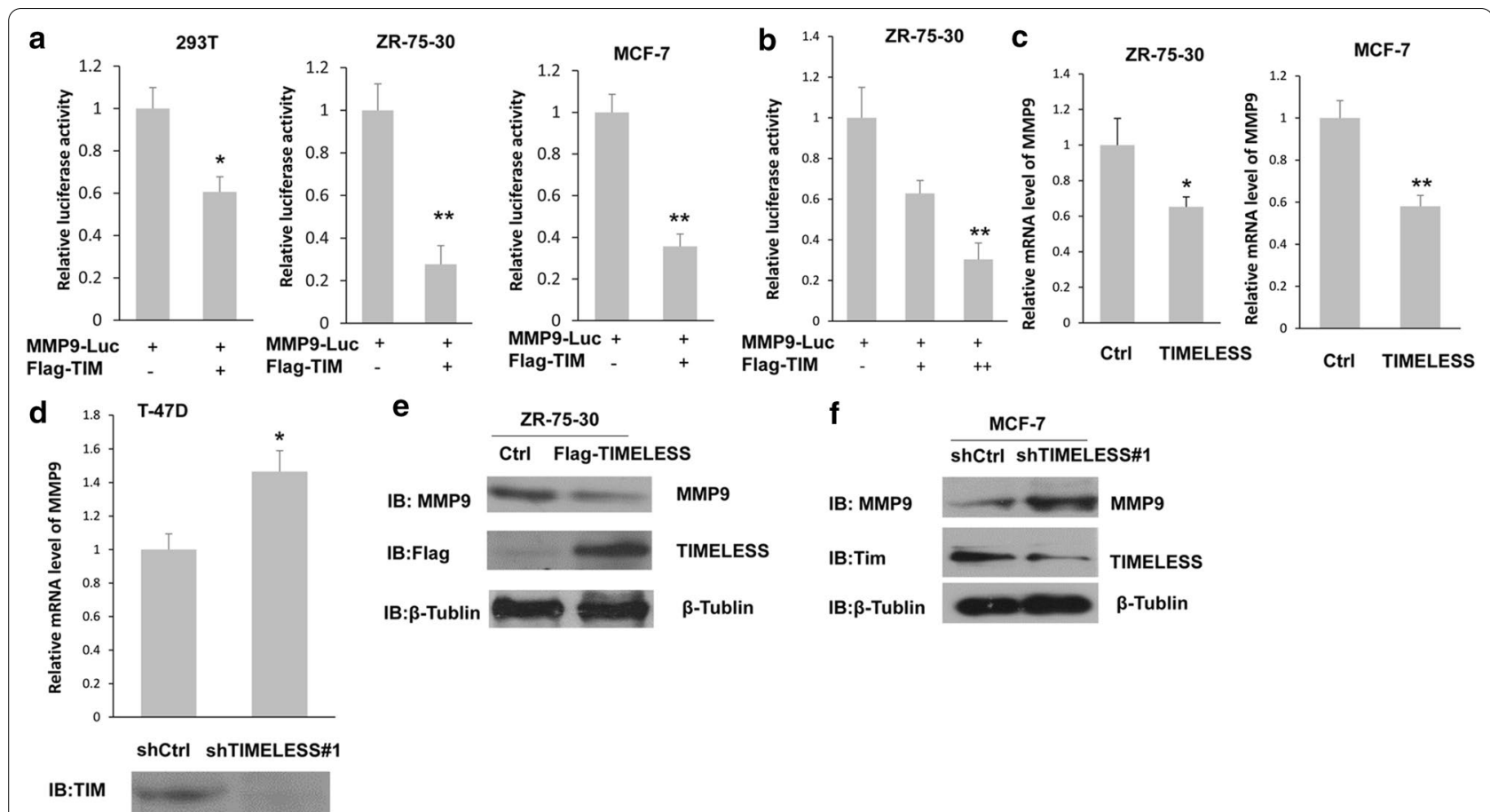

Fig. 2 The effect of TIMELESS on the expression of MMP9. a The cells were transfected with MMP9 luc with or without Flag-TIMELESS, and then cells were collected and luciferase activity was measured after $24 \mathrm{~h}$ transfection. $\mathbf{b}$ Similar experiment as A was performed but with an increasing dose of Flag-TIMELESS. c The breast cancer cells were transfected with a control vector or Flag-TIMELESS for $24 \mathrm{~h}$ and then subjected to the real-time PCR analysis. $\mathbf{d}$ T47D cells were transfected with shcontrol vector or shTIMELESS\# 1 for $24 \mathrm{~h}$, and then the mRNA level was measured by real-time PCR. $\mathbf{e}$ ZR-75-30 cells were transfected with control vector or Flag-TIMELESS for $24 \mathrm{~h}$, followed by western blot with anti-MMP9, anti-Flag or anti- $\beta$-Tubulin antibodies. $\mathbf{f M C F}-7$ cells were transfected with shcontrol vector or shTIMELESS\# 1 for $24 \mathrm{~h}$, followed by western blot with anti-MMP9, anti-TIMELESS or anti- $\beta$-Tubulin antibodies. Each bar represents the mean \pm s.d. from three independent experiments. ${ }^{\prime *}, p^{\prime \prime}<0.05 ;{ }^{\prime \prime * *{ }^{\prime \prime},} p<0.01$

binding site in the promoter of MMP9) and NF-kB-mutMMP9-luci (mutant of NF- $\mathrm{kB}$ binding site in the promoter of MMP9). The structural schematic was shown in Fig. 3c. The reporter gene assay showed that TIMELESS decreased 70\% activity of the wild-type MMP9 reporter gene, while TIMELESS only decreased $40 \%$ activity of the two mutants (Fig. 3d), indicating that TIMELESS may inhibit the expression of MMP9 through NF-kB binding site.

\section{TIMELESS interacted with NF-KB p65}

Since TIMELESS could inhibit the activation of NF-kB signaling pathway, we examined whether TIMELESS could interact with p65, which is a member of NF-kB. The immunoprecipitation experiment identified exogenous TIMELESS could interact with p65 (Fig. 4a, b). Immunoprecipitated p65 from T47D cells was detected by anti-TIMELESS antibody, indicating the interaction between TIMELESS and p65 (Fig. 4c). Through the immunofluorescence experiment, TIMELESS and p65 were mainly distributed in the nucleus. At the same time, the overexpression of TIMELESS does not affect the location of p65 in the cell (Fig. 4d). The direct interaction between TIMELESS and p65 was studied via mammalian two-hybrid systems in ZR-75-30 cells. Compared with the control group, the co-expression of TIMELESS and p65 resulted in a twofold increase of luciferase reporter gene (Fig. 4e), indicating that there is direct interaction between the two proteins in cells. This suggested that TIMELESS may participate in the NF- $\mathrm{KB}$ signal pathway via binding with $\mathrm{p} 65$.

\section{TIMELESS inhibits the acetylation of $p 65$ by inhibiting its interaction with its acetyltransferase CBP}

The acetylation of p65 is considered to be a crucial condition of activation of NF- $\mathrm{kB}$ signaling pathway, and CBP is the classical acetyltransferase of p65 [26]. Therefore, whether the overexpression of TIMELESS affects the interaction between p65 and its acetyltransferase CBP is detected. It can be seen from Fig. 5a that when TIMELESS and CBP are co-expressed, the interaction band of p65 and CBP is obviously weakened compared with the overexpression of CBP alone (Fig. 5a), indicating that TIMELESS inhibited the interaction between p65 and its acetyltransferase. The detection of the acetylation level of p65 showed that the overexpression of TIMELESS did 


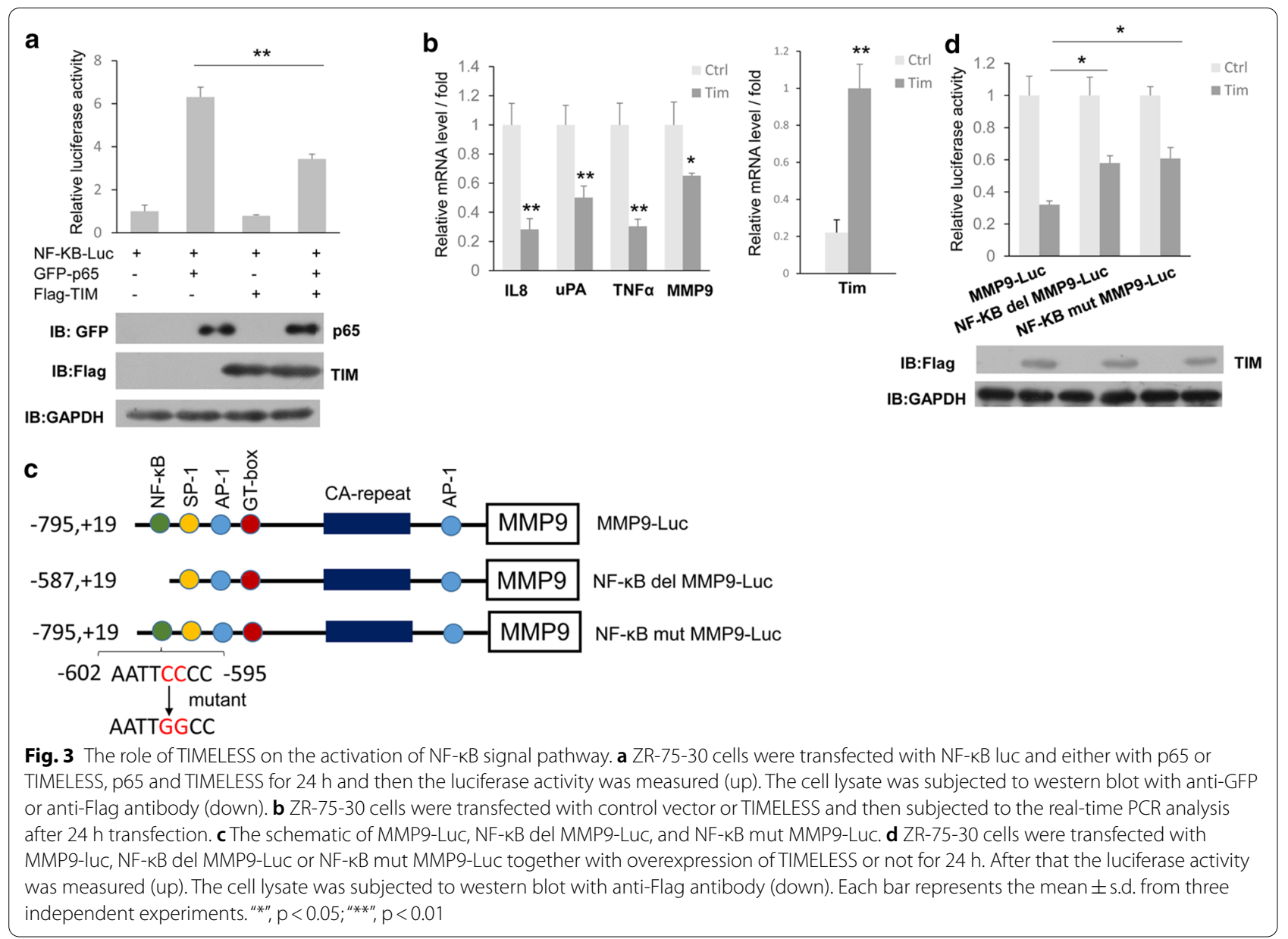

decrease the acetylation level of p65 (Fig. 5b), while the acetylation level of p65 in cells with TIMELESS knockdown was higher than that in the control group (Fig. 5c). These data indicated that TIMELESS can reduce the acetylation of p65 through inhibiting the association of the acetyltransferase CBP and p65, and then repress the activation of NF- $\mathrm{kB}$ signal pathway.

\section{Discussion}

Metastasis is the main cause of unsuccessful treatment, recurrence and death of breast cancer patients. Therefore, it is an urgent problem to search for an effective metastasis target of breast cancer. Because of the relatively high expression of MMP9 in patients with high invasive breast cancer, it is of great clinical significance to study the regulation mechanism of MMP9 expression. TIMELESS, first discovered in Drosophila, has been found in recent years to regulate various cellular functions, embryonic development, and initiation and progress of cancer, apart from regulating the circadian rhythm of organisms [27]. TIMELESS plays an important role in the development of breast cancer, mainly in its influence on the proliferation ability of breast cancer cells [28]. However, there are few reports regarding the mechanisms on TIMELESS regulating the invasive and metastatic ability of breast cancer cells. Our study reveals a new molecular regulatory mechanism, that is, TIMELESS can inhibit breast cancer migration and invasion by reducing the transcription activity of MMP9, and systematically expounds the molecular mechanism of TIMELESS regulating NF- $\mathrm{kB} /$ MMP9 pathway. It provides a theoretical basis for the clinical use of MMP9 as a target to inhibit metastasis by inducing TIMELESS.

Metastasis is a main biological characteristic of malignant tumors, and also the main cause of death. The occurrence and development of tumor not only depends on tumor cell itself but also depends on the survival environment of tumor cell, namely tumor microenvironment [29]. More and more researches focus on the role of tumor microenvironment in tumorigenesis, development, invasion and metastasis. MMPs are a group of zinc and calcium-dependent endopeptidases, which is composed of more than 20 members. It is an important protease for degradation of extracellular matrix and plays 


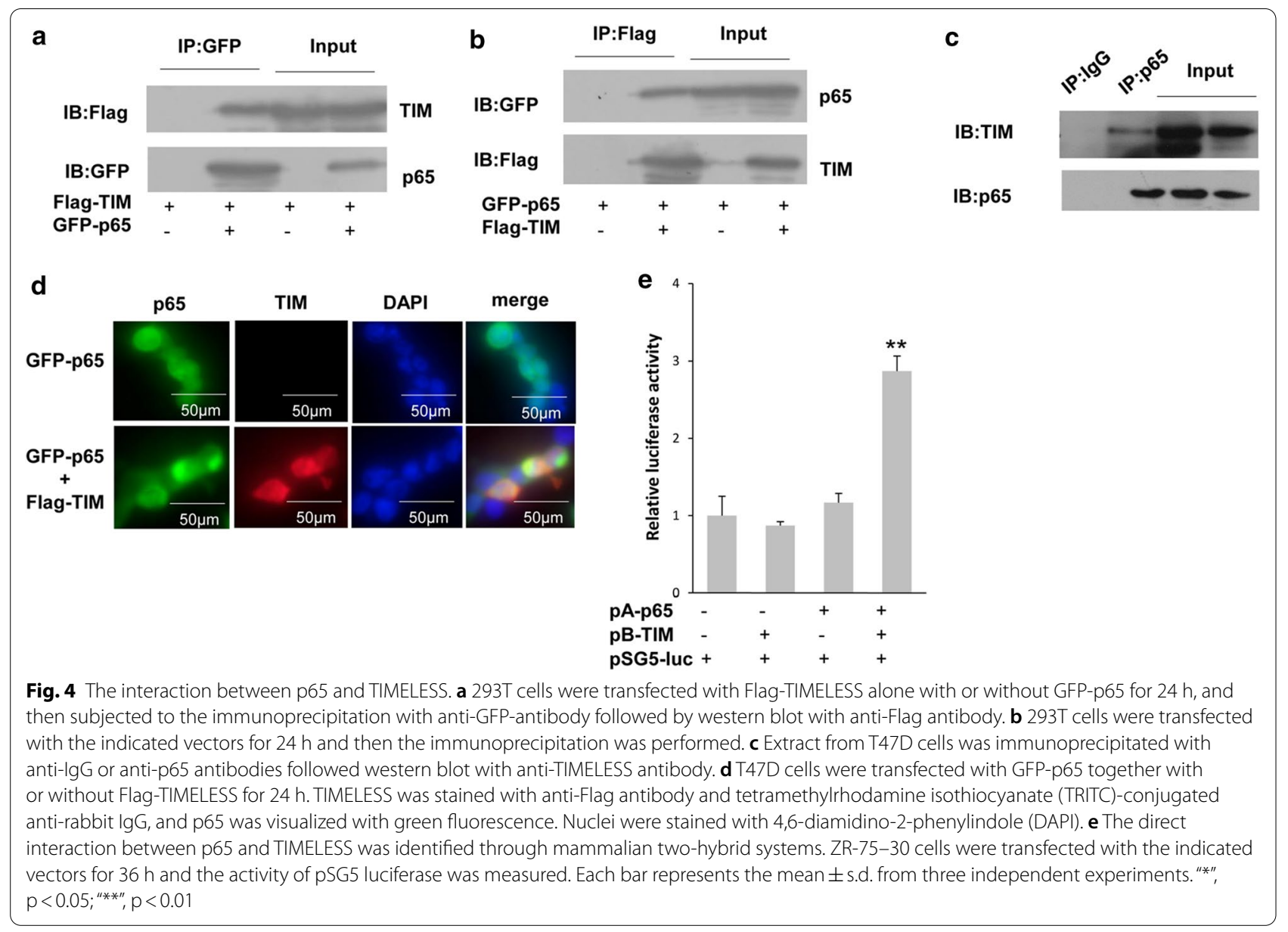

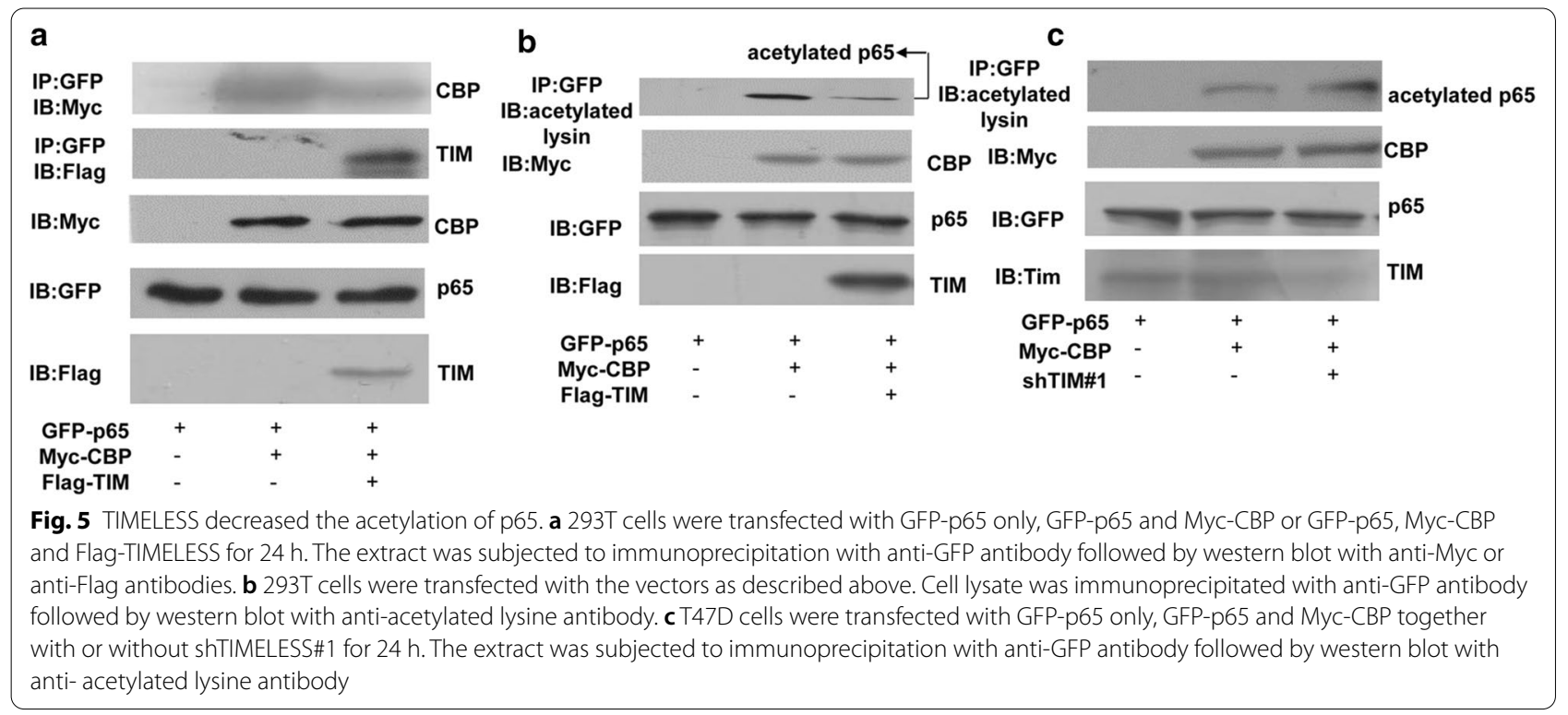


a key role in tumor proliferation, angiogenesis, invasion and metastasis. A large number of reports showed that MMPs were highly expressed in metastatic tumors. Matrix metalloproteinase-9 (MMP-9) is an important member of the MMPs family. It mainly degrades collagen IV, collagen $\mathrm{V}$ and gelatin, and plays a crucial role in the metastasis of the tumor. The expression of MMP-9 expression is positively correlated with lymph node metastasis and histological grade of endometrial carcinoma [30], and negatively correlated with invasion, metastasis and bone metastasis of breast cancer [31]. The pathological analysis of MMP-9 in primary ureteral carcinoma showed that the expression of MMP-9 was significantly higher than that in normal tissues [32].

In recent years, researchers have detected that the expression level of TIMELESS is often significantly higher or lower in cancerous tissues than that of noncancerous tissues adjacent to the tumor in various organs cancer. More studies have proved that it may be related to the progression of cancer. For example, TIMELESS can boost the development of liver cancer [33]. TIMELESS overexpression in lung cancer is associated with low patient survival [34]. TIMELESS can be used as a pathological marker in colorectal cancer [35], etc. For breast cancer, TIMELESS regulates the ribosylation of ER $\alpha$ and activates cell proliferation-related signaling pathways by acting as a novel cofactor for Er $[28]$. However, there are few studies on the regulation mechanism of invasion and metastasis in breast cancer cells by TIMELESS. Our work shows that TIMELESS can inhibit the invasion and metastasis of breast cancer cells by decreasing the expression of MMP9 (Figs. 1 and 2). This gives TIMELESS a new role in the field of the tumor and provides a basis for further revealing the fine regulatory mechanism of MMP9.

NF- $\mathrm{KB}$ pathway is involved in many pathological processes of carcinogenesis, including proliferation, cell survival, apoptosis, invasion, angiogenesis and inflammation. There are five members in NF- $\mathrm{kB}$ family, and p65/P50 complex plays an important role in NF- $\mathrm{kB}$ signal pathway. In the classical NF- $\kappa B$ signaling pathway, IKB which is the repressor of $\mathrm{p} 65 / \mathrm{p} 50$ is degraded due to ubiquitination with stimulation of activation signal, thus relieving the inhibition of $\mathrm{p} 65 / \mathrm{p} 50$ and promoting p65/p50 to play a role in transcriptional activation. Numbers of target genes are regulated by p65/ P50 such as c-myc, p53, TNF-a, COX-2, VEGF, uPA, IL-8 and MMPs, etc. [36]. Post-translational modifications of p65 confer more complexity and functional diversity on the NF- $\mathrm{B}$ pathway. Among these modifications, phosphorylation of serine-threonine residues at p65 specific sites is considered to play an important role in transcriptional activation [37]. In addition, the acetylation of p65 is also closely related to the activation of NF-kB [26]. Our results show that TIMELESS can form complex with p65 and CBP, thus inhibiting the interaction between $\mathrm{p} 65$ and its acetyltransferase CBP (Fig. 5a), and then reducing the acetylation level of p65 (Fig. 5b). The detection of NF- $\mathrm{KB}$ downstream target gene showed that TIMELESS overexpression did reduce the mRNA level of NF- $\mathrm{KB}$ downstream target gene (Fig. 3b). Therefore, TIMELESS may be a new regulatory factor of NF-kB signaling pathway and participate in the activation of NF- $\mathrm{kB}$ signaling pathway.

It has been reported that an NF- $\mathrm{kB}$ binding site localized in the promoter of MMP9 gene. The mutation or deletion of NF-KB binding site does reduce the inhibition ratio of TIMELESS to MMP9 promoter but does not completely release the inhibition of MMP9 gene transcription. This suggests that there may be some other ways for TIMELESS to down-regulate the expression of MMP-9. TIMELESS contains not only a classical TIMELESS domain but also three DDT domains [27]. The DDT domain has a DNA binding ability, indicating that TIMELESS may play a role as a transcription factor in associating with DNA localized in the promoter of MMP9. But this work needs to be further studied.

Once the tumor metastasizes, the treatment of cancer is easily out of control. Therefore, the search for new molecular targets for precise treatment has been a hot topic. In this study, a novel molecular mechanism of TIMELESS expression regulating the invasion and metastasis of breast cancer is acquired, which may provide a new basis for the treatment of metastatic breast cancer. It is expected that there will be more in-depth research in this area in the future to provide a more solid theoretical foundation for the treatment of malignant tumors.

\section{Conclusions}

In this study, we identified that TIMELESS may repress the invasion and metastasis of breast cancer cells via inhibiting the acetylation of $\mathrm{p} 65$, inhibiting the activation of NF- $\mathrm{kB}$, thus down-regulating the expression of MMP9, and then inhibiting the invasion and metastasis of breast cancer cells.

\section{Supplementary Information}

The online version contains supplementary material available at https://doi. org/10.1186/s12935-021-01752-y.

Additional file 1: Figure S1. The correlation between the expression of Timeless and the survival of patients with Basal-like breast cancer by KM analysis. The survival analysis of 239 Basal-like breast cancer patients was performed by Kaplan-Meier method, and the overall survival (OS) was the time of death or last follow-up. Comparisons on difference between groups were based on the Log-rank test, and $\mathrm{P}<0.05$ was identified as a statistically significant difference. The cut-off values involved in the results 
of data analysis are the optimal values selected for each group of highlow expression, corresponding to the optimal values of the ROC curve (receiver operating characteristic). The optimal value calculation, data analysis and picture drawing are all done in the database through Kaplan Meier-plotter database (USA).

Additional file 2: Figure S2. A and B. MTT assay evaluating the effect of TIMELESS on the proliferation of breast cancer cells. Cells were transfected with FLAG-TIMELESS and control vector (Ctrl) or shTIMELESS\#1 and control vector (shCtrl). After $24 \mathrm{~h}$, the cells were performed to the MTT assay according to the manufacturer's instructions.

Additional file 3: Figure S3. The knockdown efficiency of shTIMELESS. T47D cells were transfected with shcontrol, shTIMELESS\#1 or shTIMELESS\#2 for $24 \mathrm{~h}$, and then the cell lysis was subjected to western blot with anti-TIMELESS or GAPDH antibodies.

\section{Abbreviations}

CBP: CREB binding protein; MMP9: Matrix metalloproteinase9; PARP1: Poly (ADP-ribose) polymerase-1; DSB: DNA double-strand breaks; TNF-a: Tumor necrosis factor a; COX-2: Cyclo-oxygen-ase-2; VEGF: Vascular endothelial growth factor.

\section{Acknowledgements}

We are grateful to Professor Oliver Mortusewicz (Karolinska Institutet, Sweden) for the plasmid of PCMV-TIMELESS.

\section{Authors' contributions}

Conceived and designed the experiments: HW, SL; performed the experiments: SL, BL, LM; analyzed the data: SL, BL, LM. Contributed reagents/materials/analysis tools: BL, LM, YL, KK, YY, SA, BA; Wrote the paper: HW, SL. All authors read and approved the final manuscript.

\section{Funding}

This work was supported by Grants $(81872263,81672792$ to HW) from the National Natural Science Foundation of China and LiaoNing Revitalization Talents Program for HW.

\section{Availability of data and materials}

Please contact the corresponding author for all data requests.

\section{Ethics approval and consent to participate}

The Medical Ethics Committees of Dalian University of Technology approved this study.

\section{Consent for publication}

Not applicable.

\section{Competing interests}

The authors declare no conflict of interest.

Received: 21 February 2020 Accepted: 2 January 2021

Published online: 11 January 2021

\section{References}

1. DeSantis CE, Ma J, Gaudet MM, Newman LA, Miller KD, Goding SA, Jemal A, Siegel RL. Breast cancer statistics, 2019. CA Cancer J Clin. 2019;69(6):438-51.

2. Grundy A, Schuetz JM, Lai AS, Janoo-Gilani R, Leach S, Burstyn I, Richardson $\mathrm{H}$, Brooks-Wilson A, Spinelli JJ, Aronson KJ. Shift work, circadian gene variants and risk of breast cancer. Cancer Epidemiol. 2013;37(5):606-12.

3. Shiomi T, Okada Y. MT1-MMP and MMP-7 in invasion and metastasis of human cancers. Cancer Metas Rev. 2003;22(2-3):145-52.

4. Reimers N, Zafrakas K, Assmann V, Egen C, Riethdorf L, Riethdorf S, Berger J, Ebel S, Janicke F, Sauter G, et al. Expression of extracellular matrix metalloproteases inducer on micrometastatic and primary mammary carcinoma cells. Clin Cancer Res. 2004;10(10):3422-8.
5. Vandooren J, Van den Steen PE, Opdenakker G. Biochemistry and molecular biology of gelatinase B or matrix metalloproteinase-9 (MMP-9): the next decade. Crit Rev Biochem Mol Biol. 2013;48(3):222-72.

6. Kessenbrock K, Plaks V, Werb Z. Matrix metalloproteinases: regulators of the tumor microenvironment. Cell. 2010;141(1):52-67.

7. Yu W, Liu J, Xiong X, Ai Y, Wang H. Expression of MMP9 and CD147 in invasive squamous cell carcinoma of the uterine cervix and their implication. Pathol Res Pract. 2009;205(10):709-15.

8. Zeng ZS, Huang Y, Cohen AM, Guillem JG. Prediction of colorectal cancer relapse and survival via tissue RNA levels of matrix metalloproteinase-9. J Clin Oncol. 1996;14(12):3133-40.

9. Sillanpaa S, Anttila M, Voutilainen K, Ropponen K, Turpeenniemi-Hujanen T, Puistola U, Tammi R, Tammi M, Sironen R, Saarikoski S, et al. Prognostic significance of matrix metalloproteinase-9 (MMP-9) in epithelial ovarian cancer. Gynecol Oncol. 2007;104(2):296-303.

10. McGowan PM, Duffy MJ. Matrix metalloproteinase expression and outcome in patients with breast cancer: analysis of a published database. Ann Oncol. 2008;19(9):1566-72.

11. Sato H, Kita M, Seiki M. v-Src activates the expression of 92-kDa type IV collagenase gene through the AP-1 site and the GT box homologous to retinoblastoma control elements. A mechanism regulating gene expression independent of that by inflammatory cytokines. J Biol Chem. 1993;268(31):23460-8.

12. Gotter AL. A timeless debate: resolving TIM's noncircadian roles with possible clock function. NeuroReport. 2006;17(12):1229-33.

13. Leman AR, Noguchi E. Local and global functions of Timeless and Tipin in replication fork protection. Cell Cycle. 2012;11(21):3945-55.

14. McFarlane RJ, Mian S, Dalgaard JZ. The many facets of the Tim-Tipin protein families' roles in chromosome biology. Cell Cycle. 2010;9(4):700-5.

15. Smith-Roe SL, Patel SS, Simpson DA, Zhou YC, Rao S, Ibrahim JG, KaiserRogers KA, Cordeiro-Stone M, Kaufmann WK. Timeless functions independently of the Tim-Tipin complex to promote sister chromatid cohesion in normal human fibroblasts. Cell Cycle. 2011;10(10):1618-24.

16. Cho WH, Kang YH, An YY, Tappin I, Hurwitz J, Lee JK. Human Tim-Tipin complex affects the biochemical properties of the replicative DNA helicase and DNA polymerases. Proc Natl Acad Sci U S A. 2013;110(7):2523-7.

17. Xie S, Mortusewicz O, Ma HT, Herr P, Poon RY, Helleday T, Qian C. Timeless interacts with PARP-1 to promote homologous recombination repair. Mol Cell. 2015;60(1):163-76.

18. Young LM, Marzio A, Perez-Duran P, Reid DA, Meredith DN, Roberti D, Star A, Rothenberg E, Ueberheide B, Pagano M. TIMELESS forms a complex with PARP1 Distinct From Its Complex with TIPIN and plays a role in the DNA damage response. Cell Rep. 2015;13(3):451-9.

19. Liu Y, Ao X, Jia Z, Bai XY, Xu Z, Hu G, Jiang X, Chen M, Wu H. FOXK2 transcription factor suppresses ERalpha-positive breast cancer cell growth through down-regulating the stability of ERalpha via mechanism involving BRCA1/BARD1. Sci Rep. 2015;5:8796.

20. Bai XY, Li S, Wang M, Li X, Yang Y, Xu Z, Li B, Li Y, Xia K, Chen H, et al. Kruppel-like factor 9 down-regulates matrix metalloproteinase 9 transcription and suppresses human breast cancer invasion. Cancer Lett. 2018:412:224-35.

21. Jia Z, Wang M, Li S, Li X, Bai XY, Xu Z, Yang Y, Li B, Li Y, Wu H. U-box ubiquitin ligase PPIL2 suppresses breast cancer invasion and metastasis by altering cell morphology and promoting SNAI1 ubiquitination and degradation. Cell Death Dis. 2018;9(2):63.

22. Li S, Wang M, Ao X, Chang AK, Yang C, Zhao F, Bi H, Liu Y, Xiao L, Wu H. CLOCK is a substrate of SUMO and sumoylation of CLOCK upregulates the transcriptional activity of estrogen receptor-alpha. Oncogene. 2013;32(41):4883-91.

23. Zhao F, Wang M, Li S, Bai X, Bi H, Liu Y, Ao X, Jia Z, Wu H. DACH1 inhibits SNAI1-mediated epithelial-mesenchymal transition and represses breast carcinoma metastasis. Oncogenesis. 2015:4:e143.

24. Boggs AE, Vitolo MI, Whipple RA, Charpentier MS, Goloubeva OG, loffe OB, Tuttle KC, Slovic J, Lu Y, Mills GB, et al. alpha-Tubulin acetylation elevated in metastatic and basal-like breast cancer cells promotes microtentacle formation, adhesion, and invasive migration. Cancer Res. 2015;75(1):203-15.

25. Moreno-Bueno G, Portillo F, Cano A. Transcriptional regulation of cell polarity in EMT and cancer. Oncogene. 2008;27(55):6958-69.

26. Lecoq L, Raiola L, Chabot PR, Cyr N, Arseneault G, Legault P, Omichinski JG. Structural characterization of interactions between transactivation 
domain 1 of the p65 subunit of NF-kappaB and transcription regulatory factors. Nucleic Acids Res. 2017;45(9):5564-76.

27. Mazzoccoli G, Laukkanen MO, Vinciguerra M, Colangelo T, Colantuoni V. A timeless link between circadian patterns and disease. Trends Mol Med. 2016;22(1):68-81.

28. Magne NC, Casas GG, Docanto M, Knower KC, Young MJ, Buehn J, Sayed E, Clyne CD. Timeless is a novel estrogen receptor co-activator involved in multiple signaling pathways in MCF-7 cells. J Mol Biol. 2018;430(10):1531-43.

29. Liu Y, Ding W, Ge H, Ponnusamy M, Wang Q, Hao X, Wu W, Zhang Y, Yu W, Ao X, et al. FOXK transcription factors: regulation and critical role in cancer. Cancer Lett. 2019;458:1-12.

30. Zhang X, Xu S, Tan Q, Liu L. High expression of heparanase-2 is an independent prognostic parameter for favorable survival in gastric cancer patients. Cancer Epidemiol. 2013;37(6):1010-3.

31. Abboud-Jarrous G, Atzmon R, Peretz T, Palermo C, Gadea BB, Joyce JA, Vlodavsky I. Cathepsin $\mathrm{L}$ is responsible for processing and activation of proheparanase through multiple cleavages of a linker segment. J Biol Chem. 2008;283(26):18167-76.

32. Goldshmidt O, Nadav L, Aingorn H, Irit C, Feinstein N, Ilan N, Zamir E Geiger B, Vlodavsky I, Katz BZ. Human heparanase is localized within lysosomes in a stable form. Exp Cell Res. 2002;281(1):50-62.
33. Elgohary N, Pellegrino R, Neumann O, Elzawahry HM, Saber MM, Zeeneldin AA, Geffers R, Ehemann V, Schemmer P, Schirmacher P, et al. Protumorigenic role of Timeless in hepatocellular carcinoma. Int J Oncol. 2015:46(2):597-606.

34. Yoshida K, Sato M, Hase T, Elshazley M, Yamashita R, Usami N, Taniguchi T, Yokoi K, Nakamura S, Kondo M, et al. TIMELESS is overexpressed in lung cancer and its expression correlates with poor patient survival. Cancer Sci. 2013;104(2):171-7.

35. Wang Y, Cheng Y, Yu G, Jia B, Hu Z, Zhang L. Expression of PER, CRY, and TIM genes for the pathological features of colorectal cancer patients. Onco Targets Ther. 2016;9:1997-2005.

36. Pahl HL. Activators and target genes of Rel/NF-kappaB transcription factors. Oncogene. 1999;18(49):6853-66.

37. Ozes ON, Mayo LD, Gustin JA, Pfeffer SR, Pfeffer LM, Donner DB. NFkappaB activation by tumour necrosis factor requires the Akt serinethreonine kinase. Nature. 1999;401(6748):82-5.

\section{Publisher's Note}

Springer Nature remains neutral with regard to jurisdictional claims in published maps and institutional affiliations.
Ready to submit your research? Choose BMC and benefit from:

- fast, convenient online submission

- thorough peer review by experienced researchers in your field

- rapid publication on acceptance

- support for research data, including large and complex data types

- gold Open Access which fosters wider collaboration and increased citations

- maximum visibility for your research: over $100 \mathrm{M}$ website views per year

At BMC, research is always in progress.

Learn more biomedcentral.com/submissions 